\title{
BMJ Open Antiplatelet regimens in the long-term secondary prevention of transient ischaemic attack and ischaemic stroke: an updated network meta-analysis
}

\author{
Peng-Peng Niu, Zhen-Ni Guo, Hang Jin, Ying-Qi Xing, Yi Yang
}

To cite: Niu P-P, Guo Z-N, Jin $\mathrm{H}$, et al. Antiplatelet regimens in the long-term secondary prevention of transient ischaemic attack and ischaemic stroke: an updated network metaanalysis. BMJ Open 2016;6: e009013. doi:10.1136/ bmjopen-2015-009013

- Prepublication history and additional material is available. To view please visit the journal (http://dx.doi.org/ 10.1136/bmjopen-2015009013).

Received 8 June 2015 Revised 12 February 2016 Accepted 17 February 2016

CrossMark

Department of Neurology, Neuroscience Center, The First Hospital of Jilin University, Changchun, Jilin, China

Correspondence to Professor Yi Yang; doctoryangyi@163.com

\section{ABSTRACT}

Objective: To examine the comparative efficacy and safety of different antiplatelet regimens in patients with prior non-cardioembolic ischaemic stroke or transient ischaemic attack.

Design: Systematic review and network meta-analysis. Data sources: As on 31 March 2015, all randomised controlled trials that investigated the effects of antiplatelet agents in the long-term ( $\geq 3$ months) secondary prevention of non-cardioembolic transient ischaemic attack or ischaemic stroke were searched and identified.

Outcome measures: The primary outcome measure of efficacy was serious vascular events (non-fatal stroke, non-fatal myocardial infarction and vascular death). The outcome measure of safety was any bleeding.

Results: A total of 36 randomised controlled trials (82 144 patients) were included. Network metaanalysis showed that cilostazol was significantly more effective than clopidogrel (OR $0.77,95 \%$ credible interval $0.60-0.98)$ and low-dose (75-162 mg daily) aspirin $(0.69,0.55-0.86)$ in the prevention of serious vascular events. Aspirin (50 mg daily) plus dipyridamole (400 $\mathrm{mg}$ daily) and clopidogrel reduced the risk of serious vascular events compared with lowdose aspirin; however, the difference was not statistically significant. Furthermore, low-dose aspirin was as effective as higher daily doses. Cilostazol was associated with a significantly lower bleeding risk than most of the other regimens. Moreover, aspirin plus clopidogrel was associated with significantly more haemorrhagic events than other regimens. Direct comparisons showed similar results as the network meta-analysis.

Conclusions: Cilostazol was significantly more effective than aspirin and clopidogrel alone in the longterm prevention of serious vascular events in patients with prior non-cardioembolic ischaemic stroke or transient ischaemic attack. Cilostazol was associated with a significantly lower bleeding risk than low-dose aspirin (75-162 mg daily) and aspirin (50 mg daily) plus dipyridamole ( $400 \mathrm{mg}$ daily). Low-dose aspirin was as effective as higher daily doses. However, further large, randomised, controlled, head-to-head trials are needed, especially in non-Asian ethnic groups.

\section{Strengths and limitations of this study}

- Since the dose of aspirin ranged from 30 to $1500 \mathrm{mg}$ daily, treatment with aspirin was divided into four different regimens and treatment with aspirin plus dipyridamole was divided into two different regimens.

- Duration of follow-up was included in the model to perform the network meta-analysis.

- Estimates from sensitivity analyses were compatible with the main analysis, except that cilostazol was not significantly more effective than clopidogrel and triflusal in preventing serious vascular events when setting the prior on the variance equal to a uniform $(0,1000)$.

- Owing to the lack of data and consistent definitions, we did not differentiate between different degrees of bleeding.

- Only four small trials including 2461 Asian patients were included for the treatment of cilostazol, which decreases the confidence in the observed effect for cilostazol and means the effect of cilostazol may not be generalised to non-Asian populations.

\section{INTRODUCTION}

Cerebrovascular disease is one of the leading contributors to disease burden and results in nearly $10 \%$ of all deaths worldwide. ${ }^{1}$ Ischaemic stroke is the most common form of cerebrovascular disease. Patients who survive an ischaemic stroke or transient ischaemic attack (TIA) are at increased risk of experiencing another ischaemic stroke. On average, the annual risk for future ischaemic stroke after an initial TIA or ischaemic stroke is approximately $3-4 \% .^{2}$ Moreover, a recurrent stroke event is usually more devastating than the first stroke. ${ }^{3}$ Therefore, it is important to prevent the occurrence of future stroke among survivors of ischaemic stroke or TIA.

Antiplatelet therapy is one of the major strategies used for preventing recurrent stroke in patients who have previously 
experienced ischaemic stroke or TIA of noncardioembolic aetiology. Aspirin is currently the most widely tested antiplatelet agent and, hence, it has the most extensive evidence concerning its benefits in patients with prior ischaemic stroke and TIA. ${ }^{4}$ In addition to aspirin, there are three other antiplatelet regimens that have been approved by the US Food and Drug Administration for the secondary prevention of ischaemic stroke (ie, clopidogrel, ticlopidine, and a combination of aspirin and dipyridamole). Newer antiplatelet agents, including triflusal and cilostazol, are also potentially effective in the secondary prevention of TIA or ischaemic stroke. ${ }^{5}{ }^{6}$ However, no direct comparisons have been made among some of the antiplatelet regimens.

Network meta-analysis can assess the relative effectiveness of two treatments in cases where they have not been compared directly in a trial, but have instead each been compared with other interventions. ${ }^{7} \mathrm{We}$, therefore, performed a systematic review by using such an analytic approach to provide an up-to-date summary of the randomised controlled trials (RCTs) that have evaluated common antiplatelet agents for the secondary prevention of non-cardioembolic TIA or ischaemic stroke, and to provide an informative comparison of the relative efficacies and bleeding risk of different antiplatelet regimens.

\section{METHODS}

We followed the guidelines for conducting and reporting systematic reviews and meta-analyses. ${ }^{8}$ No protocol for this study was registered or published.

\section{Data sources and searches}

We systematically searched the EMBASE and PubMed databases for articles published up to 31 March 2015, without language or publication-type restrictions. The search was limited to studies involving human subjects. The search strategy (see online supplementary appendix 1) combined the terms 'aspirin', 'clopidogrel', 'ticlopidine', 'dipyridamole', 'cilostazol', or 'triflusal' and 'ischemic stroke' or 'transient ischemic attack'. Two of the authors (ZNG and $\mathrm{HJ}$ ) independently performed the literature search. Reference lists of systematic reviews, meta-analyses and included papers were screened. We also searched the Cochrane Central Register of Controlled Trials, ClinicalTrials.gov and Google Scholar, to identify further studies (see online supplementary appendix 1 ).

\section{Study selection}

Two authors (ZNG and YQX) independently screened the articles retrieved through the searches. The study selection was unblinded. The titles and abstracts of the retrieved articles were screened to exclude irrelevant articles. The full texts of the articles that were not excluded were retrieved and read. In the case of duplicate publications, we included papers with the largest sample size or the most complete information. Any disagreements were resolved through a discussion between the reviewers.

Studies were included if they met the following inclusion criteria: randomised, placebo-controlled or head-to-head trials; trials that investigated the efficacy of antiplatelet agents for the secondary prevention of noncardioembolic TIA or ischaemic stroke; trials that assessed the following antiplatelet agents: aspirin, clopidogrel, ticlopidine, dipyridamole, cilostazol or triflusal; trials with a long-term follow-up ( $\geq 3$ months); and trials with at least 100 patients per trial arm. ${ }^{9}$ We excluded studies that investigated the effects of other antiplatelet agents. However, for multiarm trials $(\geq 3$ arms) that involved the above antiplatelet agents and other antiplatelet agents, if we can extract two or more comparison arms that meet the criteria (including placebo), the trials will be included and the data of other antiplatelet agents will be ignored.

\section{Data extraction and quality assessment}

Data from the included articles were extracted by two independent reviewers (ZNG and $\mathrm{HJ}$ ). The following information was extracted from each study: acronym of the study or last name of the first author; publication year; country or geographical origin of the investigation; baseline characteristics of the patients; variables related to the interventions, including drug, dose and follow-up duration; number of patients in each group; and outcome data in each group. Discrepancies were resolved through a discussion between the two reviewers. Data from the intention-to-treat analysis were used.

Aspirin was the main comparison arm for many of the studies, and its dose ranged from 30 to $1500 \mathrm{mg}$ daily; treatment with aspirin was divided into the following four regimens, which were similar to those described by the Antithrombotic Trialists' Collaboration: ${ }^{4}$ aspirin 30 $50 \mathrm{mg}$ daily (very low dose), aspirin 75-162 mg daily (low dose), aspirin 283-330 mg daily (median dose) and aspirin 500-1500 mg daily (high dose). Treatment with aspirin plus dipyridamole was classified into two different regimens: aspirin $(50 \mathrm{mg})$ plus dipyridamole (400 mg) daily and aspirin (990-1300 mg) plus dipyridamole (150-300 mg) daily. The doses of other regimens were relatively consistent among most of the included studies.

The primary outcome measure of efficacy in this study was serious vascular events. The secondary outcome measure of efficacy was recurrent stroke, including both ischaemic stroke and haemorrhagic stroke. The definition of serious vascular events was as described by the Antiplatelet Trialists' Collaboration-that is, the composite of non-fatal stroke, non-fatal myocardial infarction and vascular death. ${ }^{10}$ The primary outcome measure of safety in this study was any bleeding (defined as bleeding that occurs in any part of the body). Data of discontinuation due to adverse events were reported. 
The risks of bias of the included studies were assessed unblinded by using Cochrane Collaboration's tool, ${ }^{11}$ which categorises biases according to the following key domains: selection bias (random sequence generation and allocation concealment), performance bias (blinding of participants and personnel), detection bias (blinding of outcome assessment), attrition bias (incomplete outcome data) and reporting bias (selective reporting). The risk of bias for each domain can be expressed as 'low risk', 'unclear risk', or 'high risk'. Studies with a low risk of bias in all domains are considered to have a low risk of bias, those with a high risk of bias in one or more domains are considered to have a high risk of bias and those with an unclear risk of bias in one or more domains are considered to have an unclear risk of bias. The risk of bias was assessed by two independent reviewers (HJ and YQX). Discrepancies between reviewers were resolved through a discussion between them.

\section{Data synthesis and analysis}

We performed the traditional meta-analysis with STATA, V.12.0 (Stata Corporation, College Station, Texas, USA). We measured statistical heterogeneity, using the $Q$ statistic and $\mathrm{I}^{2}$ statistic. Heterogeneity existed if the $\mathrm{Q}$ statistic was $\mathrm{p}<0.1$ or the $\mathrm{I}^{2}$ statistic was $\mathrm{I}^{2}>50 \%$. The DerSimonian and Laird random-effects model was chosen for all the analyses. ${ }^{12}$ ORs and 95\% CIs were calculated.

We performed the network meta-analysis within a Bayesian framework, using the Markov chain Monte Carlo methods in WinBUGS 1.4 (Medical Research Council Biostatistics Unit, Cambridge, UK). ${ }^{9}{ }^{13}$ The model (see online supplementary appendix 2 ) uses the number of patients experiencing an event, total number of patients and duration of follow-up, ${ }^{13}$ to estimate the ORs and corresponding 95\% credible intervals (CrIs, the Bayesian analogues of $95 \%$ CIs). ${ }^{13}{ }^{14}$ The overall residual deviance and deviance information criterion (DIC) were used both to compare fixed-effects and random-effects models of network meta-analysis and to ensure that the overall fit is adequate. The choice of the network meta-analysis model was based on the overall residual deviance and DIC. Lower values suggest a better model. ${ }^{15}$ The results are based on 100000 iterations with a burn-in of 50000 . The analysis was based on vague priors for treatment effects $\left(\theta \sim \mathrm{N}\left(0,100^{2}\right)\right)$ and between-study SD $(\tau \sim$ uniform $(0,2))$ (random-effects model), which were recommend by Dias et al. ${ }^{13}{ }^{16}$ Sensitivity to the choice of prior distribution was evaluated by setting the prior on the between-study SD to a uniform $(0,100)$ and on the variance equal to a uniform $(0,1000)$, to check the variation of the estimates. ${ }^{17}$ Convergence was assessed using the Brooks-Gelman-Rubin diagnostic tool. ${ }^{18}{ }^{19}$ Convergence was deemed to be achieved if widths of individual runs and pooled runs stabilised around a same value and their ratio was around $1 .{ }^{16} 18$
We performed a sensitivity analysis of the network meta-analysis by using the random-effects model if the fixed-effects model was chosen on the basis of the model fit. The random-effects model was then used for all of the remaining sensitivity analyses. We performed sensitivity analyses by treating two doses of aspirin (75$162 \mathrm{mg}$ daily and $283-330 \mathrm{mg}$ daily) as the same regimen. We also performed sensitivity analyses restricted to studies with a low risk of bias, those with blinded adjudication of events, those with adequate concealment of allocation, those with a low risk of attrition bias and those initiated after the year 1980; we excluded studies with follow-up duration of $<2$ years. ${ }^{9}$

The surface under the cumulative ranking curve and rankograms was used to provide a hierarchy of the regimens. ${ }^{20}$ We constructed comparison-adjusted funnel plots to examine the small study effects. ${ }^{21}$ The 'loopspecific approach' was used to evaluate inconsistencies in all closed loops of evidence formed by three (triangular loop) or four (quadratic loop) treatments within each network. ${ }^{21-23}$ We also compared the results of the consistency model with those of the inconsistency model. A comparison between the deviance and DIC statistics of the consistency and inconsistency models provides an omnibus test of consistency. ${ }^{24}$ All the analyses were performed using Stata V.12.0 and WinBUGS 1.4.

\section{RESULTS}

Figure 1 shows the flow diagram of study selection. Online supplementary appendix 3 shows the characteristics of the included studies and treatment arms. Online supplementary appendix 4 shows the data of the outcome measures. According to our search strategies, 11781 records were initially identified. Two studies were excluded because their outcome data were not available. ${ }^{2526}$ A total of 36 RCTs with 82144 patients (range, 203-20 332) were included in this meta-analysis. ${ }^{27-62}$ One of the included RCTs was retrieved by screening the references of a systematic review. ${ }^{29}$ Data of stroke or TIA subgroups of two large-scale randomised trials were extracted. ${ }^{41} 57$ There were 3 three-arm trials and 1 four-arm trial. Fifteen antiplatelet regimens were analysed, including four different aspirin regimens and two different aspirin plus dipyridamole regimens. The included studies were published between 1977 and 2013. The mean patient age at baseline was 65 years, and the mean follow-up duration was 26.9 months. A total of $11481(14.0 \%)$ serious vascular events occurred in 77 study arms, and $6568(9.5 \%)$ stroke events occurred in 70 study arms.

\section{Assessment of risk of bias for included studies}

Of the 36 studies, some were deemed to be of high or unclear risk of bias in the following domains: random sequence generation $(\mathrm{n}=3,8.3 \%)$, allocation concealment $(n=3,8.3 \%)$, blinding of participants and personnel $(n=3,8.3 \%)$, blinding of outcome assessment $(n=5$, 


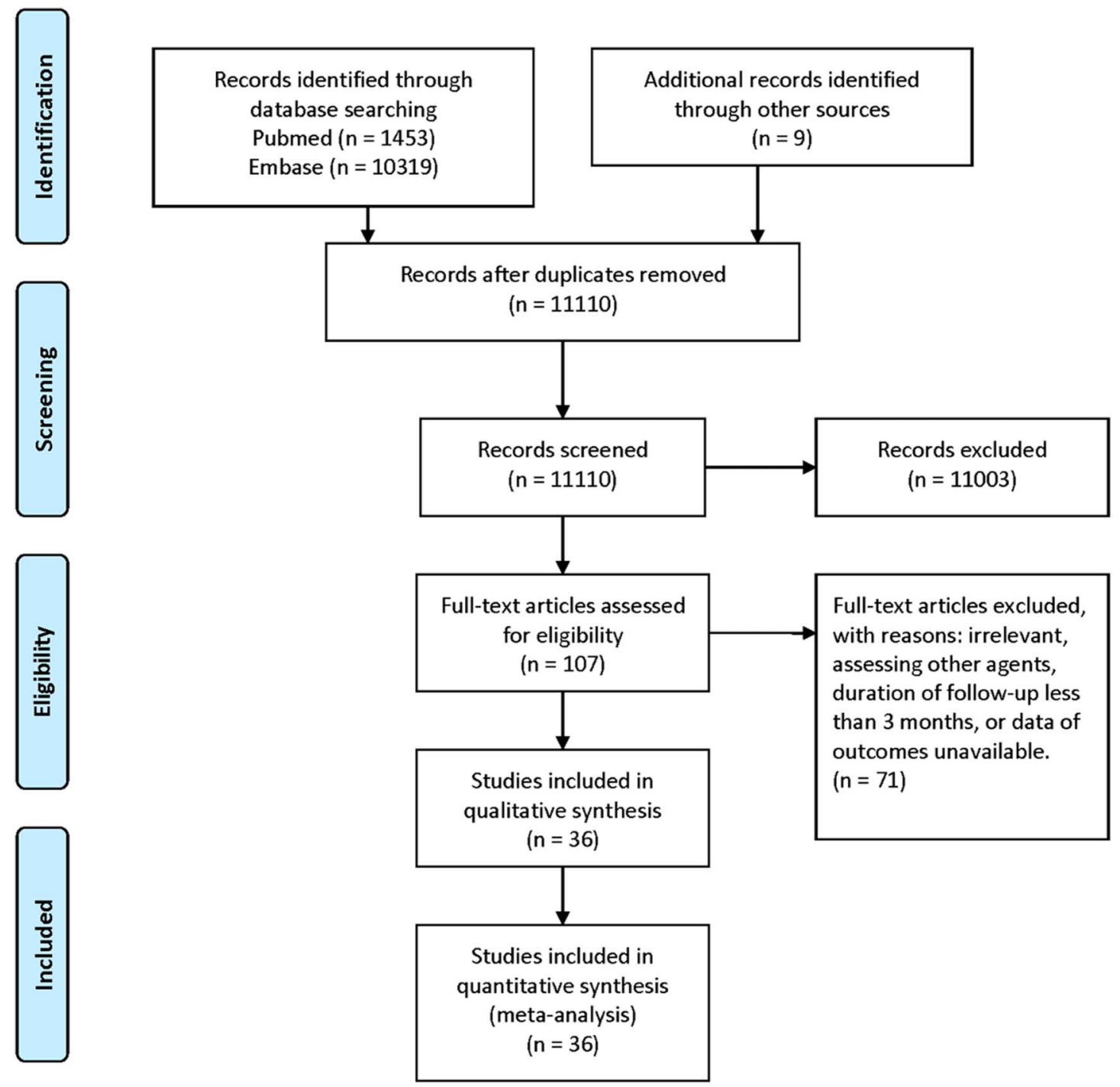

Figure 1 Flow diagram of study selection.

$13.9 \%)$, incomplete outcome reporting $(\mathrm{n}=5,13.9 \%)$ and selective reporting $(\mathrm{n}=4,11.1 \%)$. Most of the included studies $(n=29 ; 80.6 \%)$ were judged to have a low risk of bias. The risk of bias is summarised in online supplementary appendix 5 .

\section{Serious vascular events}

Figure 2 shows the network of antiplatelet regimens. Table 1, figure 3 and online supplementary appendix 6 show the results of the network meta-analysis. The random-effects model was chosen for the network meta-analysis because of a better model fit. Low, median and high doses of aspirin (75-1500 mg daily); two regimens of aspirin plus dipyridamole; clopidogrel; ticlopidine; cilostazol; and aspirin plus clopidogrel, were significantly more effective than placebo in preventing serious vascular events. Compared with very low (30$50 \mathrm{mg}$ daily), low (75-162 mg daily), median (283$330 \mathrm{mg}$ daily) and high (500-1500 mg daily) doses of aspirin, cilostazol was associated with a significant reduction of serious vascular events. The ORs $(95 \%$ CrIs) were 0.66 (0.51-0.87), 0.69 (0.55-0.86), 0.67 $(0.53-0.87)$ and $0.69(0.53-0.91)$, respectively. Cilostazol was also significantly more effective than clopidogrel (OR 0.77, 95\% CrI 0.60-0.98), ticlopidine (OR 0.71, 95\% CrI 0.54-0.94) and triflusal (OR $0.69,95 \% \mathrm{CrI}$ $0.51-0.98)$ in preventing serious vascular events. Cilostazol reduced the risk of serious vascular events when compared with aspirin ( $50 \mathrm{mg}$ daily) plus dipyridamole ( $400 \mathrm{mg}$ daily); however, the difference was not significant (OR $0.80,95 \%$ CrI 0.62-1.01). Aspirin ( $50 \mathrm{mg}$ daily) plus dipyridamole $(400 \mathrm{mg}$ daily) and clopidogrel reduced the risk of serious vascular events when compared with low-dose aspirin (75-162 mg daily); however, the differences had no statistical significance. Aspirin plus clopidogrel was significantly more effective than very low (OR $0.81,95 \%$ CrI $0.68-0.98$ ), low (OR 0.84, 95\% CrI 0.72-0.98) and median (OR $0.83,95 \%$ CrI $0.71-0.96)$ doses of aspirin, for preventing serious vascular events. There were no significant 
Figure 2 Network plot of antiplatelet regimens. The circle size is proportional to the sample size, and the line width is proportional to the sample size of each direct comparison. Next to the treatments is the sample size for each treatment. A, aspirin; $D$, dipyridamole; $\mathrm{P}$, placebo; TF, triflusal; TC, ticlopidine; CL, cilostazol; C, clopidogrel; A1, aspirin (30-50 mg) daily; A2, aspirin (75-162 mg) daily; $A 3$, aspirin (283-330 mg) daily; A4, aspirin (500-1500 mg) daily; AD1, aspirin (50 mg) plus dipyridamole (400 mg) daily; AD2, aspirin (990-1300 mg) plus dipyridamole (150-300 mg) daily.

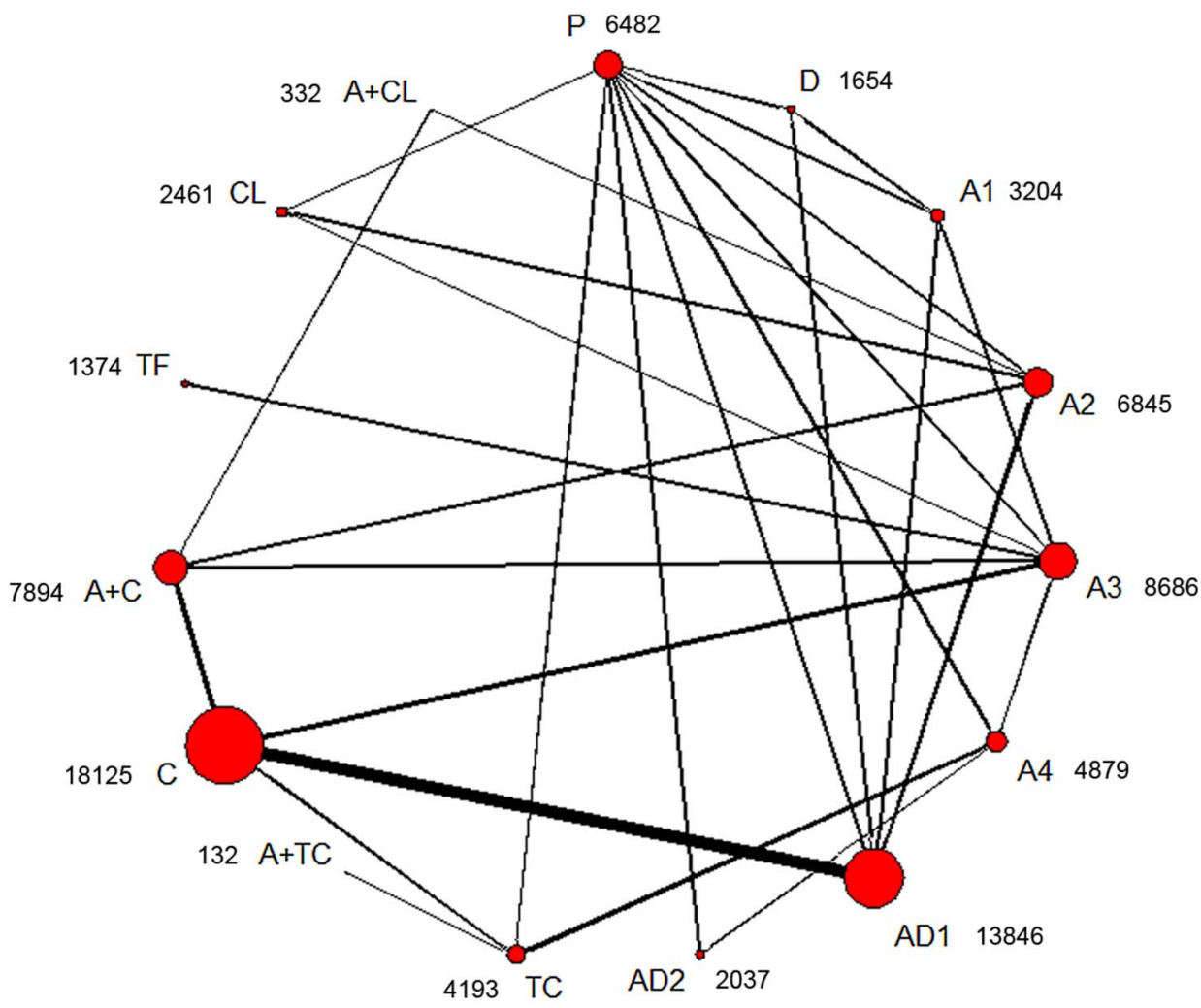

differences between different doses of aspirin in preventing serious vascular events. The results of the traditional meta-analysis were compatible with those of the network meta-analysis (table 1 and online supplementary appendix 6).

\section{Recurrent stroke}

Thirty-three trials reported recurrent stroke events. The random-effects model was chosen for the network meta-analysis because of a better model fit. Network meta-analysis showed that aspirin plus clopidogrel, two regimens of aspirin plus dipyridamole, low (75-162 mg daily) and high (500-1500 mg daily) doses of aspirin, clopidogrel, ticlopidine and cilostazol, were significantly more effective than placebo in preventing recurrent stroke (figure 3). Cilostazol was significantly more effective than ticlopidine and any dose of aspirin in preventing recurrent stroke. Cilostazol reduced the risk of stroke when compared with aspirin (50 mg) plus dipyridamole (400 mg) daily (OR 0.75, 95\% CrI 0.52-1.02) and clopidogrel (OR 0.76, 95\% CrI 0.51-1.05); however, the difference had no statistical significance. There were no significant differences between different doses of aspirin for preventing stroke. The results of the traditional meta-analysis were compatible with those of the network meta-analysis. Online supplementary appendix 7 shows the results of the network and traditional meta-analyses.

\section{Bleeding events}

Thirty trials reported bleeding events. The random-effects model was chosen for the network meta-analysis because of a better model fit. Network meta-analysis showed that four regimens of aspirin, aspirin (50 mg) plus dipyridamole (400 mg) daily, aspirin (990-1300 mg) plus dipyridamole (150-300 mg) daily, clopidogrel, ticlopidine and aspirin plus clopidogrel, were significantly associated with more haemorrhagic events than placebo (figure 3). Cilostazol was associated with more haemorrhagic events than placebo; however, the difference had no statistical significance (OR 1.18, 95\% CrI 0.80-1.79). Cilostazol was significantly associated with less haemorrhagic events than aspirin (low, median and high doses), aspirin (50 mg) plus dipyridamole (400 mg) daily and aspirin plus clopidogrel. Cilostazol was associated with less haemorrhagic events than clopidogrel (OR 0.68, 95\% CrI 0.41-1.05) and ticlopidine (OR 0.56, 95\% CrI 0.34-1.01); however, the differences had no statistical significance. Aspirin plus clopidogrel was significantly associated with more haemorrhagic events than all of the above regimens. Direct comparisons showed similar results as the network meta-analysis. Online supplementary appendix 8 shows the results of direct comparisons and network meta-analysis.

\section{Sensitivity analyses, rankings and small study effects}

The random-effects model was used for performing all the sensitivity analyses (see online supplementary appendix 6-8). The ORs were similar to those derived for the main analysis for all outcomes and regimens, and overlapping 95\% CrIs indicated that estimates from all sensitivity analyses were compatible with the main 
Table 1 Comparison of different antiplatelet regimens for the secondary prevention of serious vascular events

\begin{tabular}{|c|c|c|c|c|c|c|c|c|c|c|c|}
\hline Placebo & $\begin{array}{l}0.86 \\
(0.72-1.02)\end{array}$ & $\begin{array}{l}0.81 \\
(0.63-1.03)\end{array}$ & $\begin{array}{l}0.85 \\
(0.67-1.07)\end{array}$ & $\begin{array}{l}0.83 \\
(0.71-0.98)\end{array}$ & $\begin{array}{l}0.66 \\
(0.55-0.79)\end{array}$ & $\begin{array}{l}0.65 \\
(0.54-0.78)\end{array}$ & $\begin{array}{l}0.77 \\
(0.58-1.03)\end{array}$ & - & - & - & $\begin{array}{l}0.52 \\
(0.34-0.79)\end{array}$ \\
\hline $0.86(0.74-1.00)$ & A1 & - & $\begin{array}{l}1.05 \\
(0.86-1.27)\end{array}$ & - & $\begin{array}{l}0.77 \\
(0.64-0.93)\end{array}$ & - & - & - & - & - & - \\
\hline $0.83(0.71-0.96)$ & $\begin{array}{l}0.96 \\
(0.81-1.16)\end{array}$ & $\mathrm{A} 2$ & - & - & $\begin{array}{l}0.93^{\star} \\
(0.59-1.45)\end{array}$ & - & - & - & $\begin{array}{l}0.82 \\
(0.67-1.00)\end{array}$ & - & $\begin{array}{l}0.70 \\
(0.55-0.90)\end{array}$ \\
\hline $0.84(0.73-0.97)$ & $\begin{array}{l}0.98 \\
(0.85-1.14)\end{array}$ & $\begin{array}{l}1.02 \\
(0.87-1.19)\end{array}$ & A3 & $\begin{array}{l}0.97 \\
(0.77-1.23)\end{array}$ & - & - & - & $\begin{array}{l}0.90 \\
(0.79-1.04)\end{array}$ & $\begin{array}{l}0.86 \\
(0.68-1.08)\end{array}$ & $\begin{array}{l}0.94 \\
(0.69-1.28)\end{array}$ & $\begin{array}{l}0.65 \\
(0.23-1.85)\end{array}$ \\
\hline $0.82(0.72-0.93)$ & $\begin{array}{l}0.96 \\
(0.79-1.16)\end{array}$ & $\begin{array}{l}0.99 \\
(0.82-1.18)\end{array}$ & $\begin{array}{l}0.98 \\
(0.83-1.15)\end{array}$ & A4 & - & $\begin{array}{l}0.94 \\
(0.71-1.23)\end{array}$ & $\begin{array}{l}0.97^{*} \\
(0.71-1.32)\end{array}$ & - & - & - & - \\
\hline $0.72(0.63-0.83)$ & $\begin{array}{l}0.84 \\
(0.72-0.98)\end{array}$ & $\begin{array}{l}0.87 \\
(0.76-1.00)\end{array}$ & $\begin{array}{l}0.85 \\
(0.75-0.98)\end{array}$ & $\begin{array}{l}0.88 \\
(0.74-1.05)\end{array}$ & AD1 & - & - & $\begin{array}{l}1.00 \\
(0.93-1.09)\end{array}$ & - & - & - \\
\hline $0.70(0.59-0.83)$ & $\begin{array}{l}0.82 \\
(0.66-1.02)\end{array}$ & $\begin{array}{l}0.85 \\
(0.67-1.05)\end{array}$ & $\begin{array}{l}0.84 \\
(0.68-1.03)\end{array}$ & $\begin{array}{l}0.86 \\
(0.72-1.03)\end{array}$ & $\begin{array}{l}0.98 \\
(0.79-1.20)\end{array}$ & AD2 & - & - & - & - & - \\
\hline $0.80(0.68-0.93)$ & $\begin{array}{l}0.93 \\
(0.76-1.15)\end{array}$ & $\begin{array}{l}0.97 \\
(0.78-1.18)\end{array}$ & $\begin{array}{l}0.94 \\
(0.78-1.15)\end{array}$ & $\begin{array}{l}0.97 \\
(0.85-1.11)\end{array}$ & $\begin{array}{l}1.11 \\
(0.91-1.34)\end{array}$ & $\begin{array}{l}1.14 \\
(0.92-1.41)\end{array}$ & Ticlopidine & $\begin{array}{l}1.10 \\
(0.64-1.89)\end{array}$ & - & - & - \\
\hline $0.74(0.65-0.86)$ & $\begin{array}{l}0.86 \\
(0.74-1.03)\end{array}$ & $\begin{array}{l}0.90 \\
(0.77-1.05)\end{array}$ & $\begin{array}{l}0.88 \\
(0.78-1.01)\end{array}$ & $\begin{array}{l}0.90 \\
(0.76-1.09)\end{array}$ & $\begin{array}{l}1.03 \\
(0.93-1.16)\end{array}$ & $\begin{array}{l}1.05 \\
(0.85-1.32)\end{array}$ & $\begin{array}{l}0.93 \\
(0.77-1.14)\end{array}$ & Clopidogrel & $\begin{array}{l}0.93 \\
(0.81-1.07)\end{array}$ & - & - \\
\hline $0.70(0.59-0.83)$ & $\begin{array}{l}0.81 \\
(0.68-0.98)\end{array}$ & $\begin{array}{l}0.84 \\
(0.72-0.98)\end{array}$ & $\begin{array}{l}0.83 \\
(0.71-0.96)\end{array}$ & $\begin{array}{l}0.85 \\
(0.70-1.04)\end{array}$ & $\begin{array}{l}0.97 \\
(0.83-1.13)\end{array}$ & $\begin{array}{l}0.99 \\
(0.79-1.27)\end{array}$ & $\begin{array}{l}0.87 \\
(0.70-1.09)\end{array}$ & $\begin{array}{l}0.94 \\
(0.82-1.07)\end{array}$ & $\begin{array}{l}\text { A+ } \\
\text { Clopidogrel }\end{array}$ & - & - \\
\hline $0.82(0.63-1.04)$ & $\begin{array}{l}0.94 \\
(0.73-1.23)\end{array}$ & $\begin{array}{l}0.99 \\
(0.74-1.28)\end{array}$ & $\begin{array}{l}0.96 \\
(0.78-1.20)\end{array}$ & $\begin{array}{l}0.98 \\
(0.76-1.28)\end{array}$ & $\begin{array}{l}1.14 \\
(0.87-1.45)\end{array}$ & $\begin{array}{l}1.15 \\
(0.85-1.56)\end{array}$ & $\begin{array}{l}1.03 \\
(0.76-1.34)\end{array}$ & $\begin{array}{l}1.10 \\
(0.85-1.40)\end{array}$ & $\begin{array}{l}1.17 \\
(0.90-1.52)\end{array}$ & Triflusal & - \\
\hline $0.57(0.45-0.72)$ & $\begin{array}{l}0.66 \\
(0.51-0.87)\end{array}$ & $\begin{array}{l}0.69 \\
(0.55-0.86)\end{array}$ & $\begin{array}{l}0.67 \\
(0.53-0.87)\end{array}$ & $\begin{array}{l}0.69 \\
(0.53-0.91)\end{array}$ & $\begin{array}{l}0.80 \\
(0.62-1.01)\end{array}$ & $\begin{array}{l}0.81 \\
(0.61-1.09)\end{array}$ & $\begin{array}{l}0.71 \\
(0.54-0.94)\end{array}$ & $\begin{array}{l}0.77 \\
(0.60-0.98)\end{array}$ & $\begin{array}{l}0.81 \\
(0.63-1.05)\end{array}$ & $\begin{array}{l}0.69 \\
(0.51-0.98)\end{array}$ & Cilostazol \\
\hline \multicolumn{12}{|c|}{ 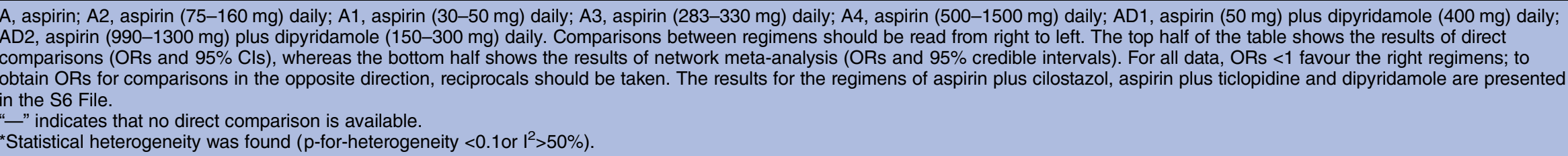 } \\
\hline
\end{tabular}


Figure 3 Estimated ORs and corresponding $95 \%$ credible intervals. Results from the network meta-analyses for different antiplatelet regimens compared with placebo are presented. The size of the squares is proportional to the statistical precision of the estimates.

Serious vascular events

OR $(95 \% \mathrm{Crl})$

Dipyridamole

Aspirin $30-50 \mathrm{mg}$ daily

Aspirin 75-162 mg daily

Aspirin 283-330 mg daily

Aspirin $500-1500 \mathrm{mg}$ daily

Aspirin $50 \mathrm{mg}$ plus dipyridamole $400 \mathrm{mg}$ daily

Aspirin 990-1300 mg plus dipyridamole $150-300 \mathrm{mg}$ daily

Ticlopidine

Aspirin plus ticlopidine

Clopidogrel

Aspirin plus clopidogrel

Triflusal

Cilostazol

Aspirin plus cilostazo

\begin{tabular}{lllll}
\cline { 2 - 5 } & & & & \\
\hline & & & & \\
\hline
\end{tabular}

Stroke

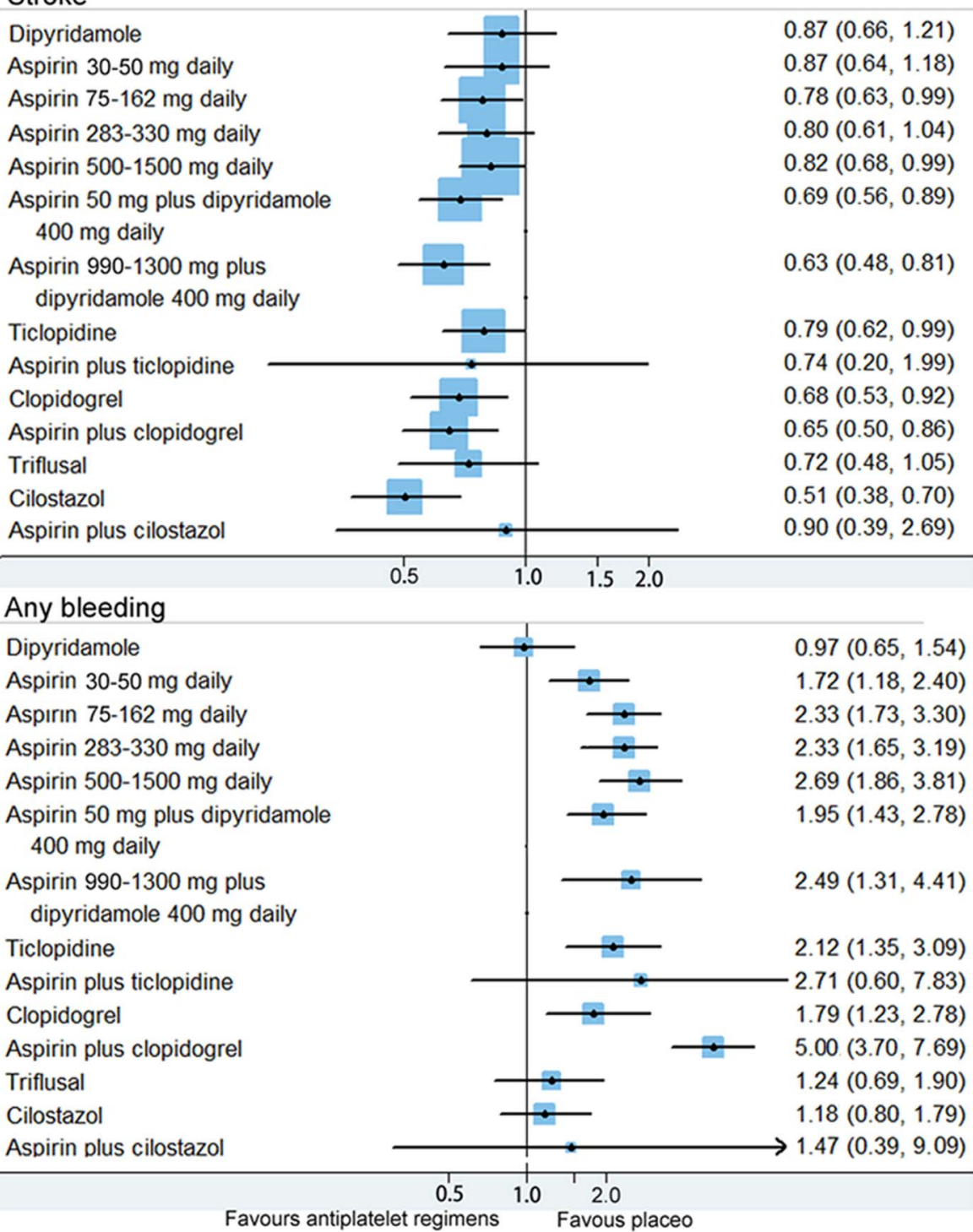

analysis. Online supplementary appendix 9 shows that the results of ORs and 95\% CrIs kept essentially unchanged for most of the comparisons when using different prior distributions. However, cilostazol was not significantly more effective than clopidogrel (OR 0.77, 95\% CrI 0.58-1.00) and triflusal (OR 0.69, 95\% CrI 
$0.50-1.01)$ in preventing serious vascular events when setting the prior on the variance equal to a uniform $(0$, 1000). Online supplementary appendix 10 presents the rankings of efficacy and safety of different regimens. Rankings of both the main and sensitivity analyses showed that cilostazol was the best option for preventing serious vascular events and recurrent stroke. Cilostazol was associated with a lower risk of bleeding than all the other effective regimens. The symmetry of comparison-adjusted funnel plots indicated that there was no evidence of small study effects (see online supplementary appendix 11).

\section{Inconsistency}

Online supplementary appendix 12 and table 2 show the inconsistencies of the test results. For outcome measures of serious vascular events and recurrent stroke, the results suggested no evidence of inconsistency in the networks. For the any bleeding outcome measure, the 'loop-specific approach' showed that the 95\% CrI (1.07-5.02) did not overlap with the value of 1 for one loop, and the mean ratio of two ORs (4.158) was large for another loop. The omnibus test also suggested evidence of inconsistency in the network of the any bleeding measure (table 2). The comparison between the deviance of the consistency and inconsistency models showed that the likely sources of inconsistency were the ECLIPse and CAIST trials. The inconsistency turned negative, and the main results of network meta-analysis were essentially unchanged after excluding these two trials. We found that these two trials had a relatively shorter follow-up (3 months), which may have led to increased heterogeneity. On the other hand, sensitivity analysis by excluding trials with $<2$ years' follow-up duration showed similar results as the main analysis for the any bleeding measure.

Table 2 Posterior summaries from random-effects consistency and inconsistency models

\begin{tabular}{|c|c|c|c|c|}
\hline \multirow{2}{*}{$\begin{array}{l}\text { Outcome } \\
\text { measures }\end{array}$} & \multicolumn{2}{|c|}{$\begin{array}{l}\text { Inconsistency } \\
\text { model }\end{array}$} & \multicolumn{2}{|c|}{$\begin{array}{l}\text { Consistency } \\
\text { model }\end{array}$} \\
\hline & Resdev & DIC & Resdev & DIC \\
\hline $\begin{array}{l}\text { Serious vascular } \\
\text { events }\end{array}$ & 76.81 & 588.566 & 71.41 & 568.622 \\
\hline Recurrent stroke & 70.59 & 516.589 & 70.07 & 507.482 \\
\hline Any bleeding & 69.6 & 441.208 & 71.73 & 436.598 \\
\hline Any bleeding ${ }^{*}$ & 66.36 & 426.594 & 65.61 & 414.301 \\
\hline
\end{tabular}

Lower values of Resdev and DIC indicate a better model fit. For serious vascular events and recurrent stroke, the consistency model had a lower Resdev and DIC, which suggests that there was no evidence of inconsistency. For the outcome of any bleeding, the inconsistency model had a lower Resdev. However, Resdev and DIC were lower in the consistency model after excluding the ECLIPse and CAIST trials.

*The ECLIPse and CAIST trials were excluded.

DIC, deviance information criterion; Resdev, posterior mean of the residual deviance.

\section{Discontinuation owing to adverse events}

Twenty-four studies reported discontinuation owing to adverse events. The random-effects network meta-analysis showed that aspirin (50 mg) plus dipyridamole (400 mg) daily was associated with a significantly higher risk of discontinuation than very low to median doses of aspirin (30-330 mg daily), and that aspirin (500-1500 mg) daily, cilostazol and ticlopidine were associated with a significantly higher risk of discontinuation than placebo. The main results of the traditional random-effects meta-analysis revealed that cilostazol was associated with a significantly higher risk of discontinuation than aspirin (75-162 mg) daily; aspirin (50 mg) plus dipyridamole (400 mg) daily was associated with a significantly higher risk of discontinuation than clopidogrel; and aspirin $50 \mathrm{mg}$ plus dipyridamole $400 \mathrm{mg}$ daily was associated with a higher risk of discontinuation than low-dose aspirin, although the difference was not significant. Online supplementary appendix 13 shows the data and results of the analyses.

\section{DISCUSSION}

In this systematic review of RCTs assessing antiplatelet regimens for the secondary prevention of TIA or ischaemic stroke, 36 trials involving 82144 patients were incorporated. The main findings of this review were that cilostazol was better than other antiplatelet regimens in the long-term secondary prevention of noncardioembolic TIA or ischaemic stroke, and that low-dose aspirin (75-162 $\mathrm{mg}$ daily) was as effective as higher daily aspirin doses.

To date, two network meta-analyses have been conducted to assess the effects of different antiplatelet regimens in the secondary prevention of non-cardioembolic TIA or ischaemic stroke. The first meta-analysis showed that using a combination of aspirin and dipyridamole was better than using thienopyridines or aspirin alone in the secondary prevention of serious vascular events after TIA or ischaemic stroke. ${ }^{63}$ The second meta-analysis mainly showed that aspirin plus dipyridamole was more protective than aspirin alone for preventing recurrent stroke after TIA or ischaemic stroke. ${ }^{64}$ The inconsistency between the results of this review and published reviews may be mainly attributed to the following factors. First, cilostazol was not included in the first review. Second, the results of the PROFESS trial were published after the first review. ${ }^{54}$ Third, some studies were not included in the second review. For example, two studies that assessed the effect of cilostazol were not included in the second review. ${ }^{44}{ }^{59}$ Lastly, the dose-response effect of aspirin was not investigated in the published reviews.

In this study, the results suggested that low-dose aspirin (75-162 mg) was as effective as higher aspirin doses for preventing serious vascular events and recurrent stroke in patients with a prior stroke or TIA, which was consist with previous studies. ${ }^{4566}$ Even though there was no statistical significance, we did find that high-dose aspirin (500-1500) was associated with a higher risk of bleeding than low-dose 
aspirin (OR 1.15, 95\% CrI 0.72-1.71). Moreover, higher dose of aspirin was associated with a higher risk of gastrointestinal side effects. ${ }^{40}$ So, it seems that low-dose aspirin is a more reasonable option than higher aspirin doses for routine long-term secondary prevention.

We believe that the protective effect of cilostazol can be ascribed not only to the antiplatelet effect but also to effects on other factors that are associated with thrombus formation. Cilostazol can increase the production of nitric oxide in human vascular endothelial cells, which can cause the dilation of blood vessels. ${ }^{67}$ Cilostazol also can inhibit smooth muscle cell proliferation, reduce intracellular calcium ion concentrations, increase plasma high-density lipoprotein-cholesterol levels, reduce plasma triglyceride levels, potentiate angiogenesis and reduce inflammation. ${ }^{67}$ These properties could potentially contribute to the prevention of secondary serious vascular events associated with this drug.

Previous studies have shown that some adverse reactions, other than haemorrhage, such as headache, palpitation, dizziness and tachycardia, occur more frequently in patients who use cilostazol. ${ }^{46}$ These adverse events may be related to vasodilatation induced by the relaxing effect of cilostazol on the vascular smooth muscle. ${ }^{68}$ This review also showed that cilostazol was associated with a significantly higher risk of discontinuation owing to adverse events than low-dose aspirin (75-162 mg daily). However, almost all of the aforementioned symptoms were typically mild or moderate, and all symptoms resolved after the discontinuation or dose tapering of cilostazol. ${ }^{46}$ Incremental increases in dose from $50 \mathrm{mg}$ might help avoid these adverse events in some patients. ${ }^{56}$

The present review has some limitations. First, the patient characteristics were heterogeneous across the trials, which is a significant limitation of this study. It is plausible that confounders such as age, sex, hypertension, diabetes mellitus and smoking, explain much of the observed effects. Second, the effects of some of the combined regimens were inconsistent with the effects of the corresponding single drugs. For example, aspirin plus cilostazol was not superior to placebo, which was inconsistent with the effect of cilostazol alone. One reason for this discrepancy may be the very small sample size of the aspirin plus cilostazol group $(n=370)$, which could have led to low statistical power. ${ }^{69}$ Third, although cardioembolic stroke was an exclusion criterion for all of the included trials, the strategies adopted in each trial to rule out cardioembolic stroke were probably suboptimal. A recent study showed that long-term monitoring with an insertable cardiac monitor was more effective than conventional follow-up for detecting atrial fibrillation after cryptogenic stroke. ${ }^{70}$ Fourth, a much more relevant safety end point such as major bleeding would be better. However, owing to the lack of data and consistent definitions, we did not differentiate between different degrees of bleeding. Fifth, we did not investigate different stroke subtypes and the severity of the cerebrovascular event because of lack of data or evidence. Sixth, a Binomial model with a cloglog link was used to approximate a Poisson process model in this study. A key assumption of this model is that in each arm of each trial the hazard is constant over the follow-up period. ${ }^{13}$ The model choice is a limitation of this study because it is unlikely that the hazard is constant in each arm of each included trial. Last, but important, the sample size and number of events for the arm of cilostazol were too small. There were only four small studies (2461 patients) with less than 200 serious vascular events, which decreases the confidence in the observed effect for cilostazol. Moreover, all trials that investigated the effect of cilostazol were performed in patients of Asian descent; therefore, the effect of cilostazol may not be generalised to non-Asian populations.

\section{CONCLUSIONS}

In conclusion, this meta-analysis, which combined sources of direct and indirect evidence, provided an overview of the efficacy and safety of different antiplatelet regimens in patients with a prior non-cardioembolic ischaemic stroke or TIA. Low-dose aspirin (75-162 mg daily), aspirin (50 mg) plus dipyridamole (400 mg) daily, clopidogrel and cilostazol can be used in the long-term secondary prevention of non-cardioembolic ischaemic stroke or TIA, among which cilostazol may be the best choice. Low-dose aspirin (75-162 mg daily) is as effective as higher daily doses. The combination of aspirin and clopidogrel significantly increases the risk of haemorrhage relative to other regimens and should not be used for routine long-term secondary prevention after an ischaemic stroke or TIA. Further large randomised, controlled head-to-head trials are needed to confirm these conclusions, especially in other ethnic groups.

Acknowledgements The authors would like to thank Editage (http://www. editage.com) for English language editing.

Contributors P-PN and YY contributed to the conception and design of the study, analysis and interpretation of the data and drafting of the work. Z-NG $\mathrm{HJ}$ and $\mathrm{Y}-\mathrm{QX}$ contributed to the design of the study, the acquisition of data and drafting of the work. All the authors approved the publication of the meta-analysis.

Funding This research received no specific grant from any funding agency in the public, commercial or not-for-profit sectors.

Competing interests None declared.

Provenance and peer review Not commissioned; externally peer reviewed.

Data sharing statement No additional data are available.

Open Access This is an Open Access article distributed in accordance with the Creative Commons Attribution Non Commercial (CC BY-NC 4.0) license, which permits others to distribute, remix, adapt, build upon this work noncommercially, and license their derivative works on different terms, provided the original work is properly cited and the use is non-commercial. See: http:// creativecommons.org/licenses/by-nc/4.0/

\section{REFERENCES}

1. Lopez AD, Mathers CD, Ezzati M, et al. Global and regional burden of disease and risk factors, 2001: systematic analysis of population health data. Lancet 2006;367:1747-57. 
2. Dhamoon MS, Sciacca RR, Rundek T, et al. Recurrent stroke and cardiac risks after first ischemic stroke: the Northern Manhattan Study. Neurology 2006;66:641-6.

3. Graham GD. Secondary stroke prevention: from guidelines to clinical practice. J Natl Med Assoc 2008;100:1125-37.

4. Antithrombotic Trialists' Collaboration. Collaborative meta-analysis of randomised trials of antiplatelet therapy for prevention of death, myocardial infarction, and stroke in high risk patients. $B M J$ 2002;324:71-86.

5. Costa J, Ferro JM, Matias-Guiu J, et al. Triflusal for preventing serious vascular events in people at high risk. Cochrane Database Syst Rev 2005;(3):CD004296.

6. Dinicolantonio $\mathrm{JJ}$, Lavie $\mathrm{CJ}$, Fares $\mathrm{H}$, et al. Meta-analysis of cilostazol versus aspirin for the secondary prevention of stroke. Am J Cardiol 2013;112:1230-4.

7. Lumley T. Network meta-analysis for indirect treatment comparisons. Stat Med 2002;21:2313-24.

8. Moher D, Liberati A, Tetzlaff J, et al. Preferred reporting items for systematic reviews and meta-analyses: the PRISMA statement. Ann Intern Med 2009;151:264-9, W64.

9. Windecker S, Stortecky S, Stefanini GG, et al. Revascularisation versus medical treatment in patients with stable coronary artery disease: network meta-analysis. BMJ 2014;348:g3859.

10. Antiplatelet Trialists' Collaboration. Collaborative overview of randomised trials of antiplatelet therapy-I: prevention of death, myocardial infarction, and stroke by prolonged antiplatelet therapy in various categories of patients. BMJ 1994;308:81-106.

11. Higgins JP, Altman DG, Gotzsche PC, et al. The Cochrane Collaboration's tool for assessing risk of bias in randomised trials. BMJ 2011;343:d5928.

12. Siontis GC, Juni $P$, Pilgrim $T$, et al. Predictors of permanent pacemaker implantation in patients with severe aortic stenosis undergoing TAVR: a meta-analysis. J Am Coll Cardiol 2014;64:129-40.

13. Dias S, Welton NJ, Sutton AJ, et al. NICE DSU Technical Support Document 2: a Generalised Linear Modelling Framework for Pairwise and Network Meta-Analysis of Randomised Controlled Trials. 2011; last updated April 2014. http://www.nicedsu.org.uk.

14. Naci $\mathrm{H}$, Brugts J, Ades T. Comparative tolerability and harms of individual statins: a study-level network meta-analysis of 246955 participants from 135 randomized, controlled trials. Circ Cardiovasc Qual Outcomes 2013;6:390-9.

15. Spiegelhalter DJ, Best NG, Carlin BP, et al. Bayesian measures of model complexity and fit. $J R$ Stat Soc: Series B (Statistical Methodology) 2002;64:583-639.

16. Giacoppo D, Gargiulo G, Aruta P, et al. Treatment strategies for coronary in-stent restenosis: systematic review and hierarchical Bayesian network meta-analysis of 24 randomised trials and 4880 patients. BMJ 2015;351:h5392.

17. Lambert PC, Sutton AJ, Burton PR, et al. How vague is vague? A simulation study of the impact of the use of vague prior distributions in MCMC using WinBUGS. Stat Med 2005;24:2401-28.

18. Brooks SP, Gelman A. General methods for monitoring convergence of iterative simulations. J Comput Graph Stat 1998;7:434-55.

19. Zhou X, Qin B, Whittington C, et al. Comparative efficacy and tolerability of first-generation and newer-generation antidepressant medications for depressive disorders in children and adolescents: study protocol for a systematic review and network meta-analysis. BMJ Open 2015;5:e007768.

20. Salanti G, Ades AE, Ioannidis JP. Graphical methods and numerical summaries for presenting results from multiple-treatment meta-analysis: an overview and tutorial. J Clin Epidemiol 2011;64:163-71.

21. Chaimani A, Higgins JP, Mavridis D, et al. Graphical tools for network meta-analysis in STATA. PLoS ONE 2013;8:e76654.

22. Donegan $\mathrm{S}$, Williamson $\mathrm{P}$, D'Alessandro U, et al. Assessing key assumptions of network meta-analysis: a review of methods. Res Synth Methods 2013;4:291-323.

23. Veroniki AA, Vasiliadis HS, Higgins JP, et al. Evaluation of inconsistency in networks of interventions. Int J Epidemiol 2013;42:332-45

24. Dias S, Welton NJ, Sutton AJ, et al. NICE DSU Technical Support Document 4: inconsistency in Networks of Evidence Based on Randomised Controlled Trials. 2011; last updated April 2014. http:// www.nicedsu.org.uk.

25. Kwon SU, Cho YJ, Koo JS, et al. Cilostazol prevents the progression of the symptomatic intracranial arterial stenosis: the multicenter double-blind placebo-controlled trial of cilostazol in symptomatic intracranial arterial stenosis. Stroke 2005;36:782-6.

26. Uchiyama S, Sakai N, Toi S, et al. Cilostazol plus aspirin versus aspirin alone in patients with symptomatic intracranial arteria stenosis: results of catharsis. J Neurol Sci 2013;333:e194.
27. Fields WS, Lemak NA, Frankowski RF, et al. Controlled trial of aspirin in cerebral ischemia. Stroke 1977:8:301-14.

28. The Canadian Cooperative Study Group. A randomized trial of aspirin and sulfinpyrazone in threatened stroke. N Engl J Med 1978;299:53-9.

29. Guiraud-Chaumeil B, Rascol A, David J, et al. [Prevention of recurrences of cerebral ischemic vascular accidents by platelet antiaggregants. Results of a 3-year controlled therapeutic trial]. Rev Neurol (Paris) 1982;138:367-85.

30. Bousser MG, Eschwege E, Haguenau M, et al. "AICLA" controlled trial of aspirin and dipyridamole in the secondary prevention of athero-thrombotic cerebral ischemia. Stroke 1983;14:5-14.

31. Sorensen PS, Pedersen H, Marquardsen J, et al. Acetylsalicylic acid in the prevention of stroke in patients with reversible cerebral ischemic attacks. A Danish cooperative study. Stroke 1983;14:15-22.

32. Tohgi $H$. The effect of ticlopidine on TIA compared with aspirin: a double-blind, twelve-month follow-up study. Agents Actions Suppl 1984;15:279-82.

33. The American-Canadian Co-Operative Study group. Persantine Aspirin Trial in cerebral ischemia. Part II: endpoint results. Stroke 1985;16:406-15.

34. A Swedish Cooperative Study. High-dose acetylsalicylic acid after cerebral infarction. Stroke 1987;18:325-34.

35. Gent M, Blakely JA, Easton JD, et al. The Canadian American Ticlopidine Study (CATS) in thromboembolic stroke. Lancet 1989;1:1215-20.

36. Hass WK, Easton JD, Adams HP Jr, et al A randomized trial comparing ticlopidine hydrochloride with aspirin for the prevention of stroke in high-risk patients. Ticlopidine Aspirin Stroke Study Group. N Engl J Med 1989;321:501-7.

37. [No authors listed]. European Stroke Prevention Study. ESPS Group. Stroke 1990;21:1122-30.

38. The Dutch TIA Trial Study Group. A comparison of two doses of aspirin (30 mg vs. $283 \mathrm{mg}$ a day) in patients after a transient ischemic attack or minor ischemic stroke. N Engl J Med 1991;325:1261-6.

39. The SALT Collaborative Group. Swedish Aspirin Low-Dose Trial (SALT) of $75 \mathrm{mg}$ aspirin as secondary prophylaxis after cerebrovascular ischaemic events. Lancet 1991;338:1345-9.

40. Farrell B, Godwin J, Richards S, et al. The United Kingdom transient ischaemic attack (UK-TIA) aspirin trial: final results. $J$ Neurol Neurosurg Psychiatr 1991:54:1044-54.

41. CAPRIE Steering Committee. A randomised, blinded, trial of clopidogrel versus aspirin in patients at risk of ischaemic events (CAPRIE). Lancet 1996;348:1329-39.

42. Diener HC, Cunha L, Forbes $\mathrm{C}$, et al. European stroke prevention study 2. Dipyridamole and acetylsalicylic acid in the secondary prevention of stroke. J Neurol Sci 1996;143:1-13.

43. Alvarez Sabin J, Matias-Guiu J, Galiano L, et al. The risk of hemorrhage in long-term treatment with aspirin and triflusal. Rev Neurol 1998;27:951-5.

44. Gotoh F, Tohgi H, Hirai S, et al. Cilostazol stroke prevention study: a placebo-controlled double-blind trial for secondary prevention of cerebral infarction. J Stroke Cerebrovasc Dis 2000;9:147-57.

45. Gorelick PB, Richardson D, Kelly M, et al. Aspirin and ticlopidine for prevention of recurrent stroke in black patients: a randomized trial. J Am Med Assoc 2003;289:2947-57.

46. Ito $\mathrm{E}$, Takahashi $\mathrm{A}$, Yamamoto $\mathrm{H}$, et al. Ticlopidine alone versus ticlopidine plus aspirin for preventing recurrent stroke. Intern Med 2003;42:793-9.

47. Matias-Guiu J, Ferro JM, Alvarez-Sabin J, et al. Comparison of triflusal and aspirin for prevention of vascular events in patients after cerebral infarction: the TACIP study: a randomized, double-blind, multicenter trial. Stroke 2003;34:840-7.

48. Culebras A, Rotta-Escalante R, Vila J, et al. Triflusal vs aspirin for prevention of cerebral infarction: a randomized stroke study. Neurology 2004;62:1073-80.

49. Diener HC, Bogousslavsky J, Brass LM, et al. Aspirin and clopidogrel compared with clopidogrel alone after recent ischaemic stroke or transient ischaemic attack in high-risk patients (MATCH): randomised, double-blind, placebo-controlled trial. Lancet 2004;364:331-7.

50. Halkes PH, van Gijn J, Kappelle LJ, et al. Aspirin plus dipyridamole versus aspirin alone after cerebral ischaemia of arterial origin (ESPRIT): randomised controlled trial. Lancet 2006;367:1665-73.

51. Kennedy J, Hill MD, Ryckborst KJ, et al. Fast assessment of stroke and transient ischaemic attack to prevent early recurrence (FASTER): a randomised controlled pilot trial. Lancet Neurol 2007;6:961-9.

52. Fukuuchi $\mathrm{Y}$, Tohgi $\mathrm{H}$, Okudera $\mathrm{T}$, et al. A randomized, double-blind study comparing the safety and efficacy of clopidogrel versus 
ticlopidine in Japanese patients with noncardioembolic cerebral infarction. Cerebrovasc Dis 2008;25:40-9.

53. Huang $\mathrm{Y}$, Cheng $\mathrm{Y}, \mathrm{Wu} \mathrm{J}$, et al. Cilostazol as an alternative to aspirin after ischaemic stroke: a randomised, double-blind, pilot study. Lancet Neurol 2008;7:494-9.

54. Sacco RL, Diener HC, Yusuf S, et al. Aspirin and extended-release dipyridamole versus clopidogrel for recurrent stroke. N Engl J Med 2008;359:1238-51.

55. Uchiyama S, Fukuuchi $\mathrm{Y}$, Yamaguchi $\mathrm{T}$. The safety and efficacy of clopidogrel versus ticlopidine in Japanese stroke patients: combined results of two Phase III, multicenter, randomized clinical trials. J Neurol 2009;256:888-97.

56. Shinohara $\mathrm{Y}$, Katayama $\mathrm{Y}$, Uchiyama S, et al. Cilostazol for prevention of secondary stroke (CSPS 2): an aspirin-controlled, double-blind, randomised non-inferiority trial. Lancet Neurol 2010;9:959-68.

57. Hankey GJ, Johnston SC, Easton JD, et al. Effect of clopidogrel plus ASA vs. ASA early after TIA and ischaemic stroke: a substudy of the CHARISMA trial. Int $J$ Stroke 2011;6:3-9.

58. Kwon SU, Hong KS, Kang DW, et al. Efficacy and safety of combination antiplatelet therapies in patients with symptomatic intracranial atherosclerotic stenosis. Stroke 2011:42:2883-90.

59. Lee YS, Bae HJ, Kang DW, et al. Cilostazol in Acute Ischemic Stroke Treatment (CAIST Trial): a randomized double-blind non-inferiority trial. Cerebrovasc Dis 2011;32:65-71.

60. Uchiyama S, Ikeda $\mathrm{Y}$, Urano $\mathrm{Y}$, et al. The Japanese Aggrenox (extended-release dipyridamole plus aspirin) stroke prevention versus aspirin programme (JASAP) study: a randomized, double-blind, controlled trial. Cerebrovasc Dis 2011;31:601-13.
61. Benavente OR, Hart RG, McClure LA, et al. Effects of clopidogrel added to aspirin in patients with recent lacunar stroke. N Engl J Med 2012;367:817-25.

62. Han SW, Lee SS, Kim SH, et al. Effect of cilostazol in acute lacunar infarction based on pulsatility index of transcranial doppler (ECLIPse): a multicenter, randomized, double-blind, placebo-controlled trial. Eur Neurol 2013;69:33-40.

63. Thijs V, Lemmens R, Fieuws S. Network meta-analysis: simultaneous meta-analysis of common antiplatelet regimens after transient ischaemic attack or stroke. Eur Heart J 2008;29:1086-92.

64. Malloy RJ, Kanaan AO, Silva MA, et al. Evaluation of antiplatelet agents for secondary prevention of stroke using mixed treatment comparison meta-analysis. Clin Ther 2013;35:1490-500 e7.

65. Patrignani $P$, Filabozzi $P$, Patrono $C$. Selective cumulative inhibition of platelet thromboxane production by low-dose aspirin in healthy subjects. J Clin Invest 1982;69:1366-72.

66. Weksler BB, Pett SB, Alonso D, et al. Differential inhibition by aspirin of vascular and platelet prostaglandin synthesis in atherosclerotic patients. N Engl J Med 1983;308:800-5.

67. Chi YW, Lavie CJ, Milani RV, et al. Safety and efficacy of cilostazol in the management of intermittent claudication. Vasc Health Risk Manag 2008;4:1197-203.

68. Yamashita K, Kobayashi S, Okada K, et al. Increased external carotid artery blood flow in headache patients induced by cilostazol. Preliminary communication. Arzneimittelforschung 1990;40:587-8.

69. Moher D, Dulberg CS, Wells GA. Statistical power, sample size, and their reporting in randomized controlled trials. JAMA 1994;272:122-4.

70. Sanna T, Diener HC, Passman RS, et al. Cryptogenic stroke and underlying atrial fibrillation. N Engl J Med 2014;370:2478-86. 